\title{
PERC rule to exclude the diagnosis of pulmonary embolism in emergency low-risk patients: study protocol for the PROPER randomized controlled study
}

Yonathan Freund ${ }^{1,2^{*}}$, Alexandra Rousseau ${ }^{3}$, France Guyot-Rousseau ${ }^{3}$, Yann-Erick Claessens ${ }^{4}$, Olivier Hugli, Olivier Sanchez ${ }^{6}$, Tabassome Simon ${ }^{1,3}$ and Bruno Riou ${ }^{1,2}$

\begin{abstract}
Background: The diagnosis of Pulmonary Embolism (PE) in the emergency department (ED) is crucial. As emergency physicians fear missing this potential life-threatening condition, PE tends to be over-investigated, exposing patients to unnecessary risks and uncertain benefit in terms of outcome. The Pulmonary Embolism Rule-out Criteria (PERC) is an eight-item block of clinical criteria that can identify patients who can safely be discharged from the ED without further investigation for PE. The endorsement of this rule could markedly reduce the number of irradiative imaging studies, ED length of stay, and rate of adverse events resulting from both diagnostic and therapeutic interventions. Several retrospective and prospective studies have shown the safety and benefits of the PERC rule for PE diagnosis in low-risk patients, but the validity of this rule is still controversial. We hypothesize that in European patients with a low gestalt clinical probability and who are PERC-negative, PE can be safely ruled out and the patient discharged without further testing.
\end{abstract}

Methods/Design: This is a controlled, cluster randomized trial, in 15 centers in France. Each center will be randomized for the sequence of intervention periods: a 6-month intervention period (PERC-based strategy) followed by a 6-month control period (usual care), or in reverse order, with 2 months of "wash-out" between the 2 periods. Adult patients presenting to the ED with a suspicion of PE and a low pre test probability estimated by clinical gestalt will be eligible. The primary outcome is the percentage of failure resulting from the diagnostic strategy, defined as diagnosed venous thromboembolic events at 3-month follow-up, among patients for whom PE has been initially ruled out.

Discussion: The PERC rule has the potential to decrease the number of irradiative imaging studies in the ED, and is reported to be safe. However, no randomized study has ever validated the safety of PERC. Furthermore, some studies have challenged the safety of a PERC-based strategy to rule-out PE, especially in Europe where the prevalence of PE diagnosed in the ED is high. The PROPER study should provide high-quality evidence to settle this issue. If it confirms the safety of the PERC rule, physicians will be able to reduce the number of investigations, associated subsequent adverse events, costs, and ED length of stay for patients with a low clinical probability of PE.

Trial registration: NCT02375919.

\footnotetext{
* Correspondence: yonathanfreund@gmail.com

${ }^{1}$ Paris Sorbonne Université, UPMC univ-Paris 6, UMRS INSERM 1166, IHU

ICAN, Paris, France

²Emergency Department, Hôpital Pitié-Salpêtrière, Assistance

Publique-Hôpitaux de Paris (APHP), Paris, France

Full list of author information is available at the end of the article
}

(c) 2015 Freund et al. Open Access This article is distributed under the terms of the Creative Commons Attribution 4.0 International License (http://creativecommons.org/licenses/by/4.0/), which permits unrestricted use, distribution, and reproduction in any medium, provided you give appropriate credit to the original author(s) and the source, provide a link to the Creative Commons license, and indicate if changes were made. The Creative Commons Public Domain Dedication waiver (http://creativecommons.org/publicdomain/zero/1.0/) applies to the data made available in this article, unless otherwise stated. 


\section{Background}

\section{Pulmonary embolism in the emergency department}

The incidence of pulmonary embolism (PE) in France and Europe has been estimated to $0.6-0.9$ per 1000 persons per year $[1,2]$. PE is a potentially lethal diagnosis [3], and its diagnosis in the Emergency Department (ED) is challenging [4].

The fear of missing this diagnosis and the poor specificity of its clinical presentation has led physicians to suspect PE in patients who present with a broad variety of symptoms such as dyspnea, chest pain, syncope, and hypotension. These patients account for more than 10 millions ED visits a year in the United States. For the last decade, the strategy for PE diagnosis has been welldefined (Fig. 1). The usual work-up for PE diagnosis first includes an assessment of the clinical probability of PE, using a structured score (the Revised Geneva Score (RGS) or Wells' score $[5,6]$ ), or an unstructured estimation of the clinical probability (referred to as the clinician "gestalt" [7-9]). In patients with low clinical probability to moderate clinical probability, sensitive Ddimer testing should be followed if positive by a Computed Tomography Pulmonary Angiogram (CTPA) in the absence of contra-indication. Patients with a high clinical probability should undergo CTPA without the need for preliminary testing. This diagnostic strategy is recommended by European guidelines [10], national expert recommendations [11] and local policies. It has been validated and is safe to exclude PE in outpatients visiting the ED [12]. However, due to its low specificity (40-60\%) [9, 13], D-dimer testing may lead to more than $50 \%$ of false positives and subsequent CTPA [9]. Furthermore, the wide availability of D-dimer testing, combined with the fear of missing PE, has led to lowering the testing threshold for suspicion of PE; hence the

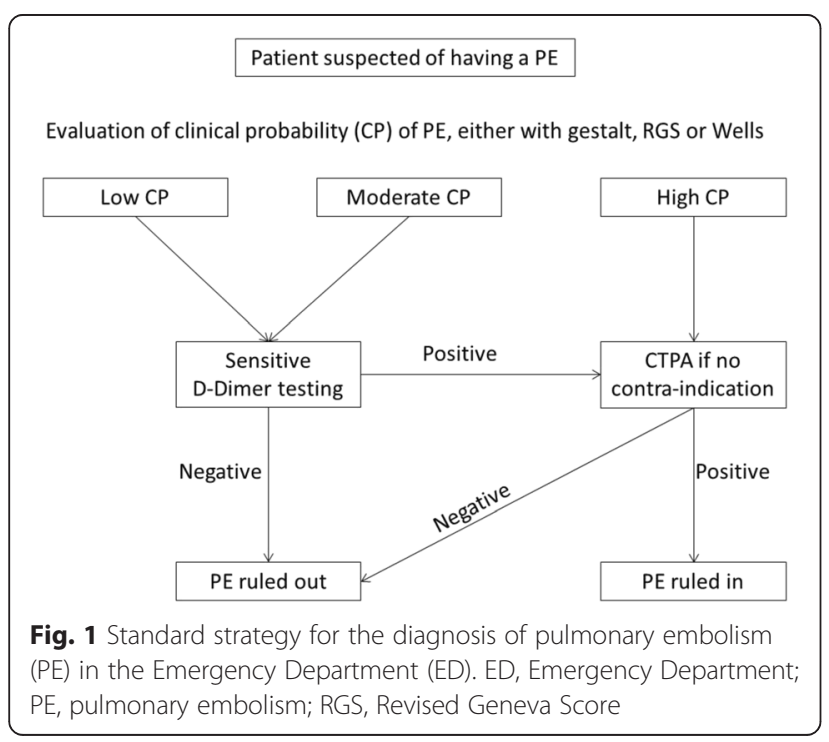

decrease in the prevalence of confirmed PE from $30 \%$ to below $10 \%$ in the United States [14-17].

Subsequently, there has been a marked rise (up to 15fold) in the utilization of CTPA in the last 15 years [18] and in the incidence of diagnosed PE [19]. However, this greater incidence of PE was not followed by a decrease in the mortality rate from $\mathrm{PE}$, but rather a global decrease in PE fatality $[19,20]$ : the prognosis of a patient with a PE improves, but the overall number of deaths from PE do not change. This suggests that PE tends to be "overdiagnosed": small PEs are more frequently diagnosed, with no clear benefit in terms of outcomes. This increased exposure to CTPA may be a source of unnecessary risks, such as contrast-induced nephropathy and allergic reactions, adverse events after anticoagulation treatment or the delayed occurrence of radiationinduced cancer [21-23].

\section{The PERC rule}

Assessing the benefit risk ratio for PE investigation, it has been calculated (using the Pausker and Kassirer method [24]) that if the pre test probability (PTP) is below $1.8 \%$, patients should not undergo D-dimer testing because a positive result would mandate a CTPA, which would have a negative benefit risk ratio [15]. To reduce the rate of unnecessary testings for PE caused by overuse of D-dimer, in 2004 Kline et al. developed a block rule of 8 binary variables (PERC rule): age < 50 years, pulse $<100 \mathrm{bpm}$, arterial oxygen saturation $\left(\mathrm{SpO}_{2}\right)>94 \%$, no unilateral leg swelling, no hemoptysis, no recent trauma or surgery, no prior PE or deep venous thrombosis (DVT) and no exogenous estrogen use [15] - PERC-negative patients are defined as fulfilling these 8 criteria. Kline et al. applied this rule in their princeps study to low-clinical-probability patients, defined by a probability of less than $15 \%$ by empirical clinical gestalt $[15,16]$. They reported that the prevalence of PE for PERC-negative patients was $1.4 \%$ (95\% confidence interval (CI) $0.5-3 \%$ ). This low rate suggests that PERC-negative patients could be safely discharged after clinical examination without further testing. Moreover, the rate of $1.4 \%$ is below the reported upper limit of false-negative rates after pulmonary angiogram or CTPA (3\%) $[12,25,26]$, which advocates for a safe alternative to further testing.

Following this princeps study, several other studies assessed the safety of a PERC-based policy to exclude PE in low-risk emergency patients with a PERC-negative rule. Two meta-analyses [17, 27] confirmed the benefits and safety of the PERC rule, with a rate of PE after follow-up lower than $1 \%$ in PERC-negative patients. They included 10 prospective and 3 retrospective studies, i.e. non-interventional studies only, accounting for a total of 14,844 ED patients with a suspicion of PE. Three 
of the 13 studies were conducted in Europe (France, Belgium and Switzerland) [28-30]. In the first 2 European studies $[28,29]$ the prevalence of PE amongst PERCnegative patients was $5.4 \%$ and $6.7 \%$, respectively (95\% CI 3-10\%). Their authors argued that the higher prevalence of PE in Europe (>20\% [17]) than in the United States $(<10 \%$ [17]) was the main reason for this lower negative predictive value, and that this rule should not be applied in high-prevalence populations. Based on the poor performance of the PERC rule, European physicians have been reluctant to apply it for excluding PE in low-risk patients. However, these two studies had several methodological bias: both studies were retrospective and did not collect PERC items prospectively. Moreover, the studied samples were not solely patients with a low gestalt clinical probability: they included unselected patients with suspected PE in the ED, with low PTP to high PTP. Although authors from one study ran a sensitivity analysis focusing on patients with low PTP, this was assessed using the Revised Geneva Score (RGS) that is based on redundant items with those of the PERC rule. These specific limits, coupled with the greater prevalence in the European studies, might explain the greater rate of false negatives. A few years later, Penaloza et al. reported that the PERC rule was safe even in Europe, when combined with a low clinical probability assessed by physician's gestalt [30], with no venous thromboembolic (VTE) event after 3-month followup. Accordingly, in a recent multicenter retrospective study, we also observed a very low prevalence of PE (0.5 \% (95 \% CI 0.1-1.1 \%)) amongst low-risk, PERCnegative ED patients [31].

Of note, all of the previously cited studies were either prospective or retrospective, but no randomized study has yet compared the benefit risk ratio of a PERC-based strategy versus the standard diagnostic strategy (Fig. 2) on occurrence of undiagnosed PE in low-risk patients.

\section{Methods}

The PROPER trial is a cluster randomized trial in France. The primary objective of this study is to assess the non-inferiority of a PERC-based diagnostic strategy for PE low-risk emergency patients, compared to the standard strategy of D-dimer testing, on the occurrence of undiagnosed VTE events. Our institutional review board authorized the study with the need for a signed informed consent from the patient (Comite de protection des personnes, Paris Ile de France 6, A00215-44).

\section{Experimental plan}

Each center will be randomized for the period: a 6month intervention period followed by a 6 -month control period, or a 6-month control period followed by a 6 month intervention period. The randomization will be prepared by URC-Est using permutation blocks (SAS 9.3, SAS Inc., Cary, NC, USA) before the first site initiation visit. The design, conduct and reporting of this study will follow the Consolidated Standards of Reporting Trials (CONSORT) statement extended to cluster randomized trials [32]. The two groups will have a different work-up for the diagnosis of PE in the ED as follows:

- Control group: standard strategy: conventional PE work-up. Every low-risk patient will undergo sensitive D-dimer testing, with subsequent CTPA if positive. In case of negative D-dimer, PE will be considered as excluded (Fig. 2a).

- Intervention group (PERC-based strategy): PE workup based on the use of the PERC rule. If all PERC criteria are negative, no further testing for PE will be recommended. If at least one criterion is positive, then the patient will undergo sensitive D-dimer testing, with subsequent CTPA if positive. In case of negative $\mathrm{D}$-dimer result, $\mathrm{PE}$ will be considered as excluded (Fig. 2b).

It has been recently reported that the cut-off for positive D-dimer should be changed for patients aged > 50 years to age $\times 10 \mathrm{ng} / \mathrm{ml}$ [33]. This strategy has been validated in a large multicenter international trial [34]. This strategy is actually endorsed by our centers. The strategy for defining "positive D-dimer" (age $\times 10 \mathrm{ng} / \mathrm{ml}$ for patients older than 50 years) will be stated before the start of the study in each center and not changed during

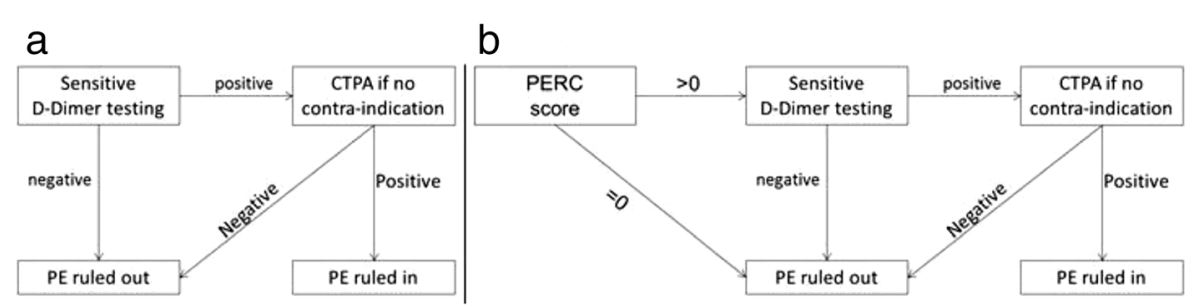

Fig. 2 Work-up for diagnosis of pulmonary embolism (PE). a Control group. b Intervention group. CTPA, Computed Tomography Pulmonary Angiogram; PE, pulmonary embolism; PERC, Pulmonary Embolism Rule-out Criteria 
the whole study period. Of note, this change will only concern patients aged 50 years or greater, i.e. with a PERC $>0$. As this will not affect PERC-negative patients, there will be no interference with our objectives.

\section{Selection of participants}

All patients with chest pain or dyspnea who attend one of the participating centers will be screened for eligibility by ED physicians and research assistants. If the treating ED physician or local investigator considers that the patient has a sufficient clinical suspicion of PE and that he needs a formal work-up for this diagnosis, and that this suspicion is low enough to discard this suspicion in case of negative D-dimer (i.e. estimated as less than $15 \%$ ), then the patient will be eligible. When a patient is eligible, his written informed consent will be obtained. In case of inability to consent, the patient will not be included in the study. The inclusion criteria are as follows:

- Patients $\geq 18$ years presenting to an ED AND

- New onset of, or worsening of shortness of breath or chest pain AND

- A low clinical probability of PE, estimated by the clinician gestalt (unstructured empirical probability of $\mathrm{PE}$ ) to be lower than $15 \%$

Exclusion criteria include the presence of any obvious cause other than a PE of symptoms, an acute severe presentation (clinical signs of respiratory distress, hypotension, $\mathrm{SpO} 2<90 \%$, shock), a contraindication to CTPA (allergy, or estimated creatinine clearance less than $30 \mathrm{ml} / \mathrm{min}$ ), pregnancy, concurrent anticoagulation treatment, current diagnosis of VTE event, inability to follow-up or to provide informed consent, lack of coverage for medical insurance, being a prisoner, and participation in another intervention trial.

After a patient is screened in the ED and may be included, the patient's emergency physician, the local investigator or a clinical research technician (CRT) will inform them of the trial, and obtain signed informed consent. After a patient is included in the study, the local investigator, the patient's emergency physicians, or the CRT will collect data on their past medical history, vital signs on admission, PERC, RGS, Wells' score, examination in the ED and discharge disposition. This will be collected on a paper Case Report Form (CRF), later entered on an electronic CRF (eCRF). Any missing data will be sought through electronic or paper records of the patients, under the supervision of the local investigator of the center.

\section{Trial objectives and outcomes}

The primary objective of this study is to assess the noninferiority of a PERC-based diagnostic strategy for ED patients with a low probability of PE based on physician's gestalt, compared to the standard strategy of Ddimer testing, on the prevalence of undiagnosed VTE events. The secondary objectives are the following:

1) to assess the reduction of unnecessary irradiative imaging studies and adverse events

2) to assess the reduction in ED length of stay

3) to assess the reduction of onset of anticoagulation regimen and associated adverse events

4) to assess the reduction of hospital admission following the ED visit, hospital readmission, and mortality at 3 months.

The primary outcome is the percentage of diagnostic strategy failure, defined as diagnosed VTE at 3-month follow-up, among patients for whom PE has been initially ruled-out. Exclusion of PE in the ED is made based upon a negative D-dimer result or a negative CTPA in both groups, or a negative PERC in the intervention group.

Follow-up will be made by telephone interview of the patient or his general practitioner, and review of any outpatient consultation or hospital visit at 3 months (13 weeks) by a CRT. The time frame of the 3-month follow-up period could be subject to minor adjustments, but should occur between day 84 and day 98 . Follow-up visit or interview will seek the occurrence of VTE events (DVT documented with ultrasonography of the lower limbs or venous computed tomography $(\mathrm{CT})$, or PE documented with positive CTPA or high probability ventilation/perfusion (V/Q) lung scan), death, return visit to the ED, and hospitalization. All medical records pertaining to the patient within this timeframe will be sought and analyzed by the local investigator, looking for reports of VTE events, or adverse events from CTPA or anticoagulation. In case of death, report of a VTE, or major cardiovascular event, the file will be analyzed by a committee of three independent experts. This method of adjudication has been described and validated in all major previous PE diagnostic studies [35, 36].

The primary criterion of a VTE event will be based on an objective diagnosis of DVT on Doppler ultrasonography, an intraluminal defect on CTPA, or a V/Q lung scan with a reported high probability. To confirm the occurrence of the primary endpoint, all files with evidence of a thromboembolic event collected by the local investigator of each center will be independently reviewed by an adjudication committee of three experts, blinded one to the other, and blinded to the study group. The adjudication committee will also review cases of death with no evidence of VTE event and will adjudicate whether or not the death is likely related to a PE. A sudden death in the absence of other obvious cause will be 
adjudicated as related to a PE. This committee will include three members, independent from the trial, with expertise in the field of $\mathrm{PE}$, and will meet the recommendations of Dechartres et al. [37].

The result of adjudication regarding the primary endpoint, and other secondary endpoints at follow-up will be entered on the eCRF by a CRT or the local investigator.

\section{Statistical analysis}

No interim analysis is planned.

Baseline characteristics of patients will be described according to group of intervention. Continuous variables will be summarized using descriptive statistics, i.e. number of subjects, mean, median, standard deviation (SD), interquartile range, minimum and maximum. Qualitative variables will be summarized by frequency and percentage. Since this is a non-inferiority study, analysis of the principal criterion will be performed on a per-protocol population. Secondary analysis will be performed based on the intention-to-treat (ITT) principle. VTE events will be defined by: DVT (assessed by proximal compression ultrasonography) or PE (a CTPA or angiography showing intraluminal defect, or a V/Q lung scan showing a high-probability pattern). The decision rule will be based on the upper bound of the $90 \% 2$-sided CI of the difference of percentage of VTE events between groups. If the upper bound of the $\mathrm{CI}$ is above the $1.5 \%$ of difference, the non-inferiority hypothesis of the intervention group will be rejected. The Dunnett and Gent chi square test will also be performed. Secondary analysis will be performed on the ITT population. Considering cluster randomization, confirmatory analysis will be performed using a generalized estimating equation (GEE) assuming an exchangeable correlation matrix structure and considering clustering at the site level. Secondary criteria will be compared under superiority hypothesis and on the ITT population. Descriptive analysis will be performed. Superiority approach will be used to compare secondary evaluation criteria between groups. The ED length stay and the mean proportion of hospital admissions following the ED visit will be compared using a mixed model, considering "center" as random effect. Unnecessary irradiative imaging, adverse events and deaths at 3 months will be compared using GEE assuming an exchangeable correlation matrix structure and considering clustering at site level. All superiority tests will be performed at $5 \%$. Pre-specified secondary analysis will include comparison of gestalt, Wells' score and RGS, and performances of the PERC35 score for patients aged under 35 years [38]. Missing data will not be replaced except for the principal criteria for the secondary ITT analysis. Missing value will be considered as an event whatever the group randomized.
According to recent large European cohorts, we estimate that the primary endpoint rate in our control group will be $1.5 \%[35,36,39]$. To be regarded as non-inferior, the maximal difference in proportions between 2 groups (Delta) should not exceed $1.5 \%$ - an absolute primary event rate of $3 \%$ in the intervention group. This failure rate corresponds to the upper bound of the observed rate after a negative CPTA and is a widely accepted criterion for the validation of diagnostic strategies for PE [40]. This rate is in line with previous landmark studies that comprise the basis of our current understanding.

\section{Sample size under non-inferiority hypothesis}

To assess non-inferiority of the "PERC strategy," with 1sided alpha $=5 \%$, beta $=20 \%, N 1=1624$ subjects are needed (East 6, Cytel, Cambridge, MA, USA). A cluster is a 6 months period for 1 site. Under the assumption of an intraclass correlation coefficient of 0.002 , an intraperiod correlation of 0.001 and a mean cluster size for one period of 60 patients, the cluster design effect would be of $D=1.118$. Considering $5 \%$ of non-evaluable subjects, with 15 sites involved in the trial, which corresponds to 30 clusters, 61 subjects per site per period are required and will lead to a total of 1920 patients.

\section{Discussion}

PE is a diagnosis that affects nearly 200,000 patients each year in France. The multiplication of diagnostic studies led to a rise in PE diagnosis, associated with a concurrent rise in the diagnosis of minor $\mathrm{PE}$, and no subsequent decrease in mortality [18-20].

According to many retrospective studies including ours, the rate of PERC-negative patients amongst patients with a low clinical probability of $\mathrm{PE}$ ranges from $15-30 \%[15,28,30,31]$. If the PERC rule was used in place of a conventional D-dimer-based diagnostic strategy, more than $10 \%$ of CTPAs could be avoided [31, 41].

Such reduction in imaging studies would be beneficial for patients. The main medical harms that can be caused by unnecessary testing for PE include adverse events from CTPA: increased risk of delayed solid tumor occurrence from irradiative imaging, and iatrogenic complications of anticoagulation for positively tested patients (either false positives or true positives for small PEs). Moreover, the benefits of diagnosing PE in low-risk patients are unclear. Mortality for patients with suspicion of PE seems very low: Kline reported that among more than 8000 patients tested for PE in the ED, only 13 patients $(0.2 \%)$ died because of $\mathrm{PE}$ (written communication).

In 2011, Newman and Schriger extrapolated the risks and benefit of D-dimer testing among a sample of 10,000 PERC-negative patients [41]. This supplemental 
testing could lead to the diagnosis of $30 \mathrm{PE}$ that would have been missed (credible interval 6-60). However, further testing in these 10,000 PERC-negative patients may cause 73 adverse events (credible interval 14-140) among which 36 fatal events (credible interval 4-69). The causes of adverse events were acute renal failure from contrast-induced nephropathy (50 per 10,000 patients), severe hemorrhage due to anticoagulation treatment (17 per 10,000 patients), and cancer resulting from radiation (5 per 10,000 patients).

Besides the estimated unfavorable medical benefit risk ratio for the patient, further testing has clear downsides: a prolonged stay in the ED, contributing to overcrowding [42, 43], overall worse short-term outcomes [44], and increased costs. In a retrospective study, median CTPA time in the ED has been reported to be $160 \mathrm{mi}-$ nutes, accounting for more than half of total ED [41]. ED length of stay could be greatly reduced if PERC was endorsed: nearly a quarter of patients with a low PTP could be discharged after just a physical examination, without the need for time-consuming biological and imaging studies.

Finally, avoiding any supplemental investigations for PERC-negative patients may also reduce the costs of ED visits, which would be of great benefit in the context of increasingly resource-stretched healthcare services. Thus, if the PERC-based strategy is shown to be noninferior to the standard strategy, it will safely and substantially reduce the volume of D-dimer and CTPA testing and, therefore, irradiation, adverse events, length of ED stay and overcrowding.

The risk for a patient recruited in the experimental group is that of a false negative PERC score, whilst the patient actually has a PE. This risk has been reported to be below $1 \%$ in previously cited meta-analyses. Furthermore, potential false-negative patients would belong to the group at lower PE risk, with an estimated 30 days mortality below $1 \%$ [45-47] - on top of the overall mortality rate estimated in the conventional group $(0.2 \%)$, the overall extrapolated added risk would be below $1 / 10,000$ at 30 days in the experimental group.

The PROPER study is a large international cluster randomized controlled trial that aims to validate the safety of the PERC rule to exclude PE in emergency patients with a low clinical probability. This trial is the first to prospectively implement and evaluate PERC in a controlled fashion to validate what previous meta-analyses have reported, and may end the controversy in this European high-prevalence population on its safety.

\section{Trial status}

Recruiting.

\section{Abbreviations}

CONSORT: Consolidated Standards of Reporting Trials; CRF: Case Report Form; CRT: clinical research technician; CT: computed tomography; CTPA: Computed Tomography Pulmonary Angiogram; DVT: deep venous thrombosis; eCRF: electronic CRF; ED: Emergency Department; GEE: generalized estimated equation; ITT: intention-to-treat; PE: pulmonary embolism; PERC: Pulmonary Embolism Rule-out Criteria; PTP: pre test probability; RGS: Revised Geneva Score; SD: standard deviation; $\mathrm{SpO}_{2}$ : arterial oxygen saturation; V/Q: ventilation/perfusion; VTE: venous thromboembolic.

\section{Competing interests}

$\mathrm{AR}, \mathrm{FGR}, \mathrm{OH}, \mathrm{YEC}, \mathrm{TS}$, and BR have no competing interests.

YF reports having received lectures fees from Lilly and BMS pharma, and travel support from Sanofi.

OS reports having received research grant support from Bayer, Daiichi Sankyo, and Portola Pharmaceuticals and fees or non-financial support for consultancy activities from Actelion, GlaxoSmithKline, Boehringer Ingelheim, and Chiesi.

YEC reports having received fees for consultancy activities from Bayer, Sanofi, Aspen and Boehringer Ingelheim.

\section{Authors' contributions}

YF conceived the study, and is the primary investigator. BR is the scientific coordinator. AR, FGR and TS handled statistical analysis and conceived the methodology of the study. YF, AR, FGR, TS and BR wrote the study protocol. $\mathrm{OS}$, YEC and $\mathrm{OH}$ provided expertise on the subject, provided substantial revisions to the protocol, and form the adjudication committee of the study. YF wrote the paper and AR, BB, TS and BR provided substantial revisions. All authors read and approved this manuscript.

\section{Acknowledgments}

The authors would like to thank Dr Bokobza for his help and support in the conception of this study.

This trial is funded by Programme Hospitalier de Recherche Clinique 2014 of the French Ministry of Health.

The funder has no role in the study design, collection, management, analysis, or interpretation of data, nor on the writing the report and decision to submit.

Other investigators of this trial has no competing interest with this study.

\section{Steering committee}

Members of the committee: Dr Yonathan Freund, Alexandra Rousseau, $\mathrm{Pr}$ Bruno Riou, Pr Tabassome Simon; Pr Patrick Ray (Paris, France),

Missions: design the study, define target population, define primary and secondary assessment criteria, and monitor inclusion rate and follow-up of the patients. All members of the steering committee will have full access to final trial dataset.

\section{Endpoint adjudication committee}

Members of the committee: Pr Olivier Hugli (Lausanne, Switzerland), Pr Olivier Sanchez (Paris, France), Pr Yann-Eric Claessens (Monaco, MC),

Missions: independently adjudicate the occurrence of likely thromboembolic event after 3 month follow-up, in case of undocumented suspicion, or death. Operating methods: for all patients who had an event during the 3 months follow-up that could be related to a thromboembolic event, the medical record will be anonymized and blinded to the study period, and sent for external adjudication to the endpoint adjudication committee.

As the research has been classified as "minimal risk," the presence of a data safety monitor board is waived. All severe adverse events will be notified to the sponsor.

\section{Author details}

${ }^{1}$ Paris Sorbonne Université, UPMC univ-Paris 6, UMRS INSERM 1166, IHU ICAN, Paris, France. ${ }^{2}$ Emergency Department, Hôpital Pitié-Salpêtrière, Assistance Publique-Hôpitaux de Paris (APHP), Paris, France. ${ }^{3}$ Plateforme de recherche clinique de l'est parisien (URCEST-CRCEST), Hôpital St Antoine, APHP, Paris, France. ${ }^{4}$ Emergency Department, Princess Grace Hospital, Monte Carlo, Monaco. ${ }^{5}$ Emergency Department, Lausanne University Hospital, Lausanne, Switzerland. ${ }^{6}$ Pneumology and Intensive Care Unit, Hôpital Européen Georges Pompidou, APHP, Université Paris Descartes, Sorbonne Paris Cite, Paris, France. 
Received: 29 July 2015 Accepted: 6 November 2015

\section{Published online: 25 November 2015}

\section{References}

1. Oger E. Incidence of venous thromboembolism: a community-based study in Western France. EPI-GETBP Study Group. Groupe d'Etude de la Thrombose de Bretagne Occidentale. Thromb Haemost. 2000;83:657-60.

2. Cohen AT, Agnelli G, Anderson FA, Arcelus Jl, Bergqvist D, Brecht JG, et al. Venous thromboembolism (VTE) in Europe. The number of VTE events and associated morbidity and mortality. Thromb Haemost. 2007;98:756-64.

3. Goldhaber SZ, Visani L, De Rosa M. Acute pulmonary embolism: clinical outcomes in the International Cooperative Pulmonary Embolism Registry (ICOPER). Lancet. 1999:353:1386-9.

4. American College of Emergency Physicians Clinical Policies Committee. Clinical Policies Committee Subcommittee on Suspected Pulmonary Embolism: clinical policy: critical issues in the evaluation and management of adult patients presenting with suspected pulmonary embolism. Ann Emerg Med. 2003;41:257-70.

5. Le Gal G, Righini M, Roy P-M, Sanchez O, Aujesky D, Bounameaux H, et al. Prediction of pulmonary embolism in the emergency department: the revised Geneva score. Ann Intern Med. 2006;144:165-71.

6. Wells PS, Anderson DR, Rodger M, Ginsberg JS, Kearon C, Gent M, et al. Derivation of a simple clinical model to categorize patients' probability of pulmonary embolism: increasing the models utility with the SimpliRED Ddimer. Thromb Haemost. 2000;83:416-20.

7. Lucassen W, Geersing G-J, Erkens PMG, Reitsma JB, Moons KGM, Büller H, et al. Clinical decision rules for excluding pulmonary embolism: a metaanalysis. Ann Intern Med. 2011;155:448-60.

8. Penaloza A, Verschuren F, Meyer G, Quentin-Georget S, Soulie C, Thys F, et al. Comparison of the unstructured clinician gestalt, the Wells score, and the revised Geneva score to estimate pretest probability for suspected pulmonary embolism. Ann Emerg Med. 2013;62:117-24. e2.

9. Chunilal SD, Eikelboom JW, Attia J, et al. Does this patient have pulmonary embolism? JAMA. 2003;290:2849-58.

10. Torbicki A, Perrier A, Konstantinides S, Agnelli G, Galiè N, Pruszczyk P, et al. Guidelines on the diagnosis and management of acute pulmonary embolism: the Task Force for the Diagnosis and Management of Acute Pulmonary Embolism of the European Society of Cardiology (ESC). Eur Heart J. 2008:29:2276-315.

11. Robert-Ebadi H, Righini M. Diagnosis of pulmonary embolism. Rev Mal Respir. 2010;28:790-99.

12. Perrier A, Roy P-M, Aujesky D, Chagnon I, Howarth N, Gourdier A-L, et al. Diagnosing pulmonary embolism in outpatients with clinical assessment, D-dimer measurement, venous ultrasound, and helical computed tomography: a multicenter management study. Am J Med. 2004;1 16:291-9.

13. Righini M, Nendaz M, Le Gal G, Bounameaux H, Perrier A. Influence of age on the cost-effectiveness of diagnostic strategies for suspected pulmonary embolism. J Thromb Haemost JTH. 2007;5:1869-77.

14. Le Gal G, Bounameaux H. Diagnosing pulmonary embolism: running after the decreasing prevalence of cases among suspected patients. J Thromb Haemost JTH. 2004;2:1244-6.

15. Kline JA, Mitchell AM, Kabrhel C, Richman PB, Courtney DM. Clinical criteria to prevent unnecessary diagnostic testing in emergency department patients with suspected pulmonary embolism. J Thromb Haemost JTH. 2004;2:1247-55.

16. Kline JA, Courtney DM, Kabrhel C, Moore CL, Smithline HA, Plewa MC, et al. Prospective multicenter evaluation of the pulmonary embolism rule-out criteria. J Thromb Haemost JTH. 2008;6:772-80.

17. Singh B, Parsaik AK, Agarwal D, Surana A, Mascarenhas SS, Chandra S. Diagnostic accuracy of pulmonary embolism rule-out criteria: a systematic review and meta-analysis. Ann Emerg Med. 2012;59:517-20. e1-4.

18. Wiener RS, Schwartz LM, Woloshin S. When a test is too good: how CT pulmonary angiograms find pulmonary emboli that do not need to be found. BMJ. 2013;347:f3368-8.

19. Wiener RS, Schwartz LM, Woloshin S. Time trends in pulmonary embolism in the United States: evidence of overdiagnosis. Arch Intern Med. 2011;171:831-7.

20. Schissler AJ, Rozenshtein A, Kulon ME, Pearson GDN, Green RA, Stetson PD, et al. CT pulmonary angiography: increasingly diagnosing less severe pulmonary emboli. PLoS ONE. 2013;8, e65669.
21. Einstein AJ, Henzlova MJ, Rajagopalan S. Estimating risk of cancer associated with radiation exposure from 64-slice computed tomography coronary angiography. JAMA J Am Med Assoc. 2007;298:317-23.

22. Mitchell AM, Kline JA. Contrast nephropathy following computed tomography angiography of the chest for pulmonary embolism in the emergency department. J Thromb Haemost JTH. 2007;5:50-4.

23. Cochran ST, Bomyea K, Sayre JW. Trends in adverse events after IV administration of contrast media. AJR Am J Roentgenol. 2001;176:1385-8.

24. Pauker SG, Kassirer JP. The threshold approach to clinical decision making. N Engl J Med. 1980;302:1109-17.

25. Stein PD, Fowler SE, Goodman LR, Gottschalk A, Hales CA, Hull RD, et al. Multidetector computed tomography for acute pulmonary embolism. N Engl J Med. 2006;354:2317-27.

26. van Beek EJ, Brouwerst EM, Song B, Stein PD, Oudkerk M. Clinical validity of a normal pulmonary angiogram in patients with suspected pulmonary embolism - a critical review. Clin Radiol. 2001;56:838-42.

27. Singh B, Mommer SK, Erwin PJ, Mascarenhas SS, Parsaik AK. Pulmonary embolism rule-out criteria (PERC) in pulmonary embolism-revisited: a systematic review and meta-analysis. Emerg Med J EMJ. 2012.

28. Hugli O, Righini M, Le Gal G, Roy P-M, Sanchez O, Verschuren F, et al. The pulmonary embolism rule-out criteria (PERC) rule does not safely exclude pulmonary embolism. J Thromb Haemost JTH. 2011;9:300-4.

29. Righini M, Le Gal G, Perrier A, Bounameaux H. More on: clinical criteria to prevent unnecessary diagnostic testing in emergency department patients with suspected pulmonary embolism. J Thromb Haemost JTH. 2005;3:188-9. author reply 190-191.

30. Penaloza A, Verschuren F, Dambrine S, Zech F, Thys F, Roy P-M. Performance of the Pulmonary Embolism Rule-out Criteria (the PERC rule) combined with low clinical probability in high prevalence population. Thromb Res. 2012;129:e189-193.

31. Bokobza J, Aubry A, Nakle N, Vincent-Cassy C, Pateron D, Devilliers C, et al. Pulmonary Embolism Rule-out Criteria vs D-dimer testing in low-risk patients for pulmonary embolism: a retrospective study. Am J Emerg Med. 2014;32:609-13.

32. Campbell MK, Elbourne DR, Altman DG. CONSORT statement: extension to cluster randomised trials. BMJ. 2004;328:702-8.

33. Douma RA, le Gal G, Söhne M, Righini M, Kamphuisen PW, Perrier A, et al. Potential of an age adjusted D-dimer cut-off value to improve the exclusion of pulmonary embolism in older patients: a retrospective analysis of three large cohorts. BMJ. 2010;340:C1475.

34. Righini M, Van Es J, Exter PLD, Roy P-M, Verschuren F, Ghuysen A, et al. Age-adjusted D-dimer cutoff levels to rule out pulmonary embolism: the ADJUST-PE study. JAMA J Am Med Assoc. 2014;311:1117-24.

35. Righini M, Le Gal G, Aujesky D, Roy P-M, Sanchez O, Verschuren F, et al. Diagnosis of pulmonary embolism by multidetector $\mathrm{CT}$ alone or combined with venous ultrasonography of the leg: a randomised non-inferiority trial. The Lancet. 2008;371:1343-52.

36. Perrier A, Roy P-M, Sanchez O, Le Gal G, Meyer G, Gourdier A-L, et al. Multidetector-row computed tomography in suspected pulmonary embolism. N Engl J Med. 2005;352:1760-8.

37. Dechartres A, Boutron I, Roy C, Ravaud P. Inadequate planning and reporting of adjudication committees in clinical trials: recommendation proposal. J Clin Epidemiol. 2009;62:695-702.

38. Mongan J, Kline J, Smith-Bindman R. Age and sex-dependent trends in pulmonary embolism testing and derivation of a clinical decision rule for young patients. Emerg Med J EMJ. 2015.

39. Righini M, Aujesky D, Roy P, et al. Clinical usefulness of $d$-dimer depending on clinical probability and cutoff value in outpatients with suspected pulmonary embolism. Arch Intern Med. 2004;164:2483-7.

40. Kruip MJHA, Leclercq MGL, van der Heul C, Prins MH, Büller HR. Diagnostic strategies for excluding pulmonary embolism in clinical outcome studies. A systematic review. Ann Intern Med. 2003;138:941-51.

41. Crichlow A, Cuker A, Mills AM. Overuse of computed tomography pulmonary angiography in the evaluation of patients with suspected pulmonary embolism in the emergency department. Acad Emerg Med Off J Soc Acad Emerg Med. 2012;19:1219-26.

42. Kline JA. Written communication. 2014.

43. Newman DH, Schriger DL. Rethinking testing for pulmonary embolism: less is more. Ann Emerg Med. 2011;57:622-7. e3.

44. Forero R, Hillman KM, McCarthy S, Fatovich DM, Joseph AP, Richardson DB. Access block and ED overcrowding. Emerg Med Australas EMA. 2010;22:119-35. 
45. Forero R, McCarthy S, Hillman K. Access block and emergency department overcrowding. Crit Care. 2011;15:216.

46. Guttmann A, Schull MJ, Vermeulen MJ, Stukel TA. Association between waiting times and short term mortality and hospital admission after departure from emergency department: population based cohort study from Ontario, Canada. BMJ. 2011;342:d2983.

47. Jiménez D, Yusen RD, Otero R, Uresandi F, Nauffal D, Laserna E, et al. Prognostic models for selecting patients with acute pulmonary embolism for initial outpatient therapy. Chest. 2007;132:24-30.

Submit your next manuscript to BioMed Central and take full advantage of:

- Convenient online submission

- Thorough peer review

- No space constraints or color figure charges

- Immediate publication on acceptance

- Inclusion in PubMed, CAS, Scopus and Google Scholar

- Research which is freely available for redistribution 